\title{
Structural insights into the galanin receptors signaling
}

3 Authors and Affiliations:

4 Wentong Jiang ${ }^{1,2}$, Sanduo Zheng ${ }^{1,3^{*}}$

$5 \quad{ }^{1}$ National Institute of Biological Sciences, Beijing, China

$6{ }^{2}$ Graduate School of Peking Union Medical College, Beijing, China

$7{ }^{3}$ Tsinghua Institute of Multidisciplinary Biomedical Research, Tsinghua University, Beijing,

8 China

\section{Abstract}

11 Galanin is a biologically active neuropeptide, and functions through three distinct G

12 protein-coupled receptors (GPCRs), namely GALR1, GALR2 and GLAR3. GALR signaling

13 plays important roles in regulating various physiological processes such as energy

14 metabolism, neuropathic pain, epileptic activity, and sleep homeostasis. GALR1 and GALR3

15 signal through the $G_{i / o}$ pathway, whereas GALR2 signals mainly through the $G_{q / 11}$ pathway.

16 However, the molecular basis for galanin recognition and G protein selectivity of GALRs

17 remains poorly understood. Here, we report the cryoelectron microscopy structures of the

18 GALR1-G and the GALR2-G $\mathrm{G}_{\mathrm{q}}$ complexes bound to the endogenous ligand galanin or spexin.

19 The galanin peptide mainly adopts an alpha helical structure, which binds at the extracellular

20 vestibule of the receptors, nearly parallel to the membrane plane without penetrating deeply

21 into the receptor core. Structural analysis combined with functional studies reveals important

22 structural determinants for the G protein selectivity of GALRs as well as other class A

23 GPCRs. In addition, we show that the zinc ion is a negative allosteric regulator of GALR1 but

24 not GALR2. Our studies provide insight into the mechanisms of G protein selectivity of

25 GPCRs and highlight potential novel function of the neuromodulator zinc ion as a modulator

26 of GPCR signaling in the central nervous system. 


\section{Significance Statement}

30

31 Galanin exerts various physiological functions through galanin receptors, including

32 antinociceptive activity, depression and sleep. Here, we reveal a distinct binding site and

33 binding pose of galanin peptide in galanin receptors from that of the published structures of

34 peptide-bound GPCRs. Moreover, our work show that the neuromodulator zinc ion

35 negatively modulates galanin signaling in the central nervous system, and further advances

36 our understanding of mechanisms of G protein selectivity of GPCRs. These unique features

37 of galanin receptors can be exploited for rational design of subtype selective ligands for

38 treatments of neurological disorders. 


\section{Introduction}

40 Galanin is a 29 or 30 -amino-acid peptide that was isolated from pig intestine in 1983 (1).

41 Through its wide distribution in the nervous system and the endocrine system, galanin is

42 involved in a variety of physiological functions, including regulation of hormones and

43 neurotransmitters release, antinociceptive activity, depression and sleep/wake homeostasis

$44(2,3)$. The endogenous action of galanin is mediated through activation of three distinct

45 receptor subtypes (GALR1-3), which belong to the class A of G protein-coupled receptors

46 (GPCRs) family $(4,5)$.

48 GALR subtypes vary in their downstream signaling pathways and the tissue distribution.

49 GALR1 and GLAR3 mainly couple to the inhibitory $\mathrm{Ga}_{\mathrm{i} / \mathrm{o}}$ pathway, leading to the inhibition of

50 the adenylyl cyclase activity and the decrease of the intracellular adenosine 3',5'-cyclic

51 monophosphate (cAMP) level. By contrast, GALR2 mainly couples to the stimulatory

52 pathway of $\mathrm{G}_{\mathrm{q} / 11}$, inducing the formation of inositol triphosphate (IP3), which in turn

53 increases the cytosolic $\mathrm{Ca}^{2+}$ level (3) (Fig. 1A). While GALR1 is particularly enriched in the

54 nervous system, GALR2 and GALR3 are broadly distributed in brain as well as peripheral

55 tissues. GALRs activation via overexpression or administration of galanin in the nervous

56 system of animals suppresses seizure development and neuropathic pain behavior, and

57 show anxiolytic and antidepressant effect (6-10). A missense mutation in galanin peptide

58 was identified as a cause of temporal lobe epilepsy (TLE) (11). Moreover, galanin expression

59 is upregulated in the injured neurons, and galanin has been shown to play a role in

60 neuroprotection and neuronal regeneration (12, 13). Accumulating evidence indicate that

61 GALRs signaling is a key regulator of both sleep time and sleep/awake homeostasis in

62 model organisms such as zebrafish and mouse $(14,15)$. Therefore, GALRs are potential

63 therapeutic targets for the treatment of pain, epilepsy, depression, neuron injury and sleep

64 disorders.

65

66 In addition to galanin, the endogenous galanin-like peptide (GALP) and spexin have been 
67 shown to activate GALRs $(16,17)$. Although these peptides share high sequence similarity,

68 they show distinct receptor binding preference. In contrast to galanin that interacts with all

69 three receptor subtypes, spexin specifically activate GALR2 and GALR3. Homology

70 modeling and site directed mutagenesis studies revealed the essential residues of galanin

71 involved in the receptor binding and activation, and the potential galanin binding site of

72 GALRs $(18,19)$. However, the molecular details of galanin binding and the peptide

73 selectivity of GALRs remain poorly defined at the molecular level. Moreover, little is known

74 about the molecular basis of G protein coupling specificty of GALRs. To gain insight into the

75 molecular basis of ligand recognition and ligand selectivity of GALRs and extend our

76 understanding of $\mathrm{G}$ protein selectivity, we sought to determine the cryoelectron microscopy

77 (cryo-EM) structures of GALR1 and GARL2 in complex with $\mathrm{G}_{0}$ and $\mathrm{G}_{\mathrm{q}}$ heterotrimer

78 respectively. 
81 Results

\section{Structure determination}

83 To obtain stable GPCR-G protein complexes, we used engineered thermostable mini-G

84 proteins, which only contain the GTPase domain of Ga but still bind to Gßy heterodimer and

85 recapitulate the pharmacological and structural changes in GPCRs induced by the full-length

86 Ga proteins (20). Moreover, the $\mathrm{N}$-terminal residues of $\mathrm{aN}$ in mini-Gao and mini-Gaq were

87 replaced by the equivalent residues of Gai to acquire the ability to bind the antibody

88 fragment scFv16 that stabilizes the nucleotide-free GPCR-G protein complex (21).

89 Furthermore, we introduced a linker that contains a 3C protease cleavage site, between the

90 C-terminus of the receptor and the N-terminus of the mini-Ga to create a GPCR-G fusion

91 protein. The GALR1-mini-Gao or GALR2-mini-Gaq fusion protein was transiently expressed

92 in Expi293 cell, and was assembled with purified Gß1Y2 and scFv16 in the presence of

93 galanin (SI Appendix, Fig. S1). The resulting GALR1-mini-Go complexes were co-eluted

94 and mono-disperse with or without $3 \mathrm{C}$ protease treatment from the size exclusion

95 chromatography, indicating that GALR1 forms a stable complex with mini-Go (SI Appendix,

96 Figs. S1A-1C). The peak fraction corresponding to the complexes were concentrated and

97 subjected to cryo-EM single particle analysis. 2D class average analysis showed that the

98 GALR1-mini-G fusion protein complex gives more orientations than the GALR1-mini-G 99 complex without a linker between the receptor and min-Ga (SI Appendix, Figs. S1D and

100 S1E). Combination of the two datasets enables us to obtain a final cryo-EM map of the 101 GALR1-mini-Go complex at a global nominal resolution of $3.3 \AA$ (SI Appendix, Fig. S2 and 102 Table S1). The structure of the galanin- and spexin-bound GALR2-minGq fusion complex 103 was determined to a nominal resolution of $3.3 \AA$ and $3.5 \AA$, respectively (SI Appendix, Fig. 104 S3 and Table S1). The high quality EM map allowed us to unambiguously assign side 105 chains of the galanin peptide 1-17 and the most amino acids of the receptors except the 106 extreme terminal residues and some intracellular loops because of their high flexibility (Fig. $107 \mathbf{1} \boldsymbol{B}$ and $\mathbf{C})$. The overall structure of the GALR1-Go complex resembles that of the 108 GALR2-Gq complex, with root-mean-square deviation values of $0.886 \AA$ for the Ca atoms of 
109 the receptors and $0.604 \AA$ for the $\mathrm{Ca}$ atoms of the $\mathrm{G}$ proteins.

\section{Comparison of galanin binding pockets of GALR1 and GALR2}

112 The existence of bulky aromatic amino acids and the high quality EM density map allowed 113 us to unambiguously assign side chains of galanin (Fig. $\mathbf{2 A}$ ). The $\mathrm{N}$-terminal portion of 114 galanin (residue 1-15) was well resolved due to its direct contact with the receptors, which is 115 consistent with previous studies showing that the binding affinity of $\mathrm{N}$-terminal region of 116 galanin (1-16) for the receptors is comparable to the full-length galanin $(22,23)$. Moreover, 117 the N-terminal region (1-16) but not the remaining part is highly conserved in GALP and 118 spexin peptides (SI Appendix, Fig. S4A), both of which are able to activate the receptors, 119 further supporting our structural observation. Galanin mainly forms an alpha helical structure 120 when bound to the receptor as well as in solution itself $(24,25)$. It occupies at the 121 extracellular vestibule of GALRs that is equivalent to the binding site of a positive allosteric 122 agonist LY2119620 in M2R (26) (SI Appendix, Fig. S4B). It lays on top of the receptor, 123 nearly parallel to the membrane plane and distant from the toggle switch $W^{6.48}$, the 124 conformational change of which is essential for the receptor activation. By contrast, most 125 neuropeptide agonists of class A GPCRs such as endothelin, orexin and opioid peptides 126 binds nearly perpendicular to the membrane plane with one end buried in the helical cavity 127 and the other end interacting with the extracellular loops, and these peptides penetrate in 128 proximity to the toggle switch (27-29) (SI Appendix, Fig. S4B). Galanin contacts all seven 129 TM helices as well as extracellular loops ECL2 and ECL3, burying a surface area of $866 \AA^{2}$, 130 which accounts for the high affinity binding of galanin for GALRs in the sub-nanomolar range 131 (30). GALR1 and GALR2 use overlapping but distinct set of residues to contact galanin, 132 mostly via hydrophobic and hydrogen bond interactions (Fig. 2). The first N-terminal residue 133 of G1 lies between TM1 and TM7, and is closer to TM1 of GALR1 than that of GALR2 (Fig. $134 \mathbf{2 F}$ ), which may explain that removal of $\mathrm{G} 1$ in galanin reduced its binding affinity for GALR1 135 but not GALR2 (24). W2 is sandwiched between L277 ${ }^{\text {ELC3 }}$ and F282 ${ }^{7.32}$ of GALR1 and 136 makes additional hydrogen bond with $S 281^{7.31}$ (Fig. 2C). Therefore, mutation of W2 in 
137 galanin or F282 $2^{7.32}$ results in significant loss of binding for the receptors $(18,31)$. F282 ${ }^{7.32} \mathrm{~A}$

138 mutation in GALR1 almost abolished galanin potency (Fig. 2G), and F271 ${ }^{7.32} \mathrm{~A}$ mutation in 139 GALR2 reduced galanin potency by nearly 100-fold (Fig. 2H). A7E mutation that was 140 identified as a cause of TLE disease likely causes a clash with nearby hydrophobic residues, 141 accounting for reduced binding affinity for GALRs (11) (Fig. 2C). Y9 penetrates into the 142 receptor core, about $10 \AA$ above the toggle switch (SI Appendix, Fig. S4B), and is 143 hydrogen-bonded by $Q 92^{2.61}$ in GALR1 or Q82 2.61 in GALR2 (Figs. 2 C and E). Mutation of 144 Q92 2.61 or Q82 $2^{2.61}$ to alanine reduced galanin potency by almost 100 -fold (Figs. 2 G and $\boldsymbol{H}$ ). 145 Our structural observation is also consistent with previous studies showing that W2 and Y9 146 are vital for galanin binding to the receptors (31). However, because of distinct residues of 147 ECL2 and ECL3 involved in binding galanin, the conformations of these regions are different 148 between GALR1 and GALR2 (Fig. 2F). For instance, V274 in the ECL3 of GALR1 engages 149 hydrophobic interaction with L11 (Fig. 2C), while the equivalent residue in GALR2, Q263 150 rotates away from galanin due to its longer side chain and hydrophilic nature, resulting in the 151 conformational change of ECL3 (Fig. 2F). As a result, V274G mutation reduced agonist 152 potency by about 370-fold, while Q263A mutation showed little effect (Figs. 2 G and $\boldsymbol{H}$ ). 153 ECL2 forms an antiparallel $\beta$-sheet, which is a characteristic of peptide receptors. It covers 154 galanin as a lid-like structure and forms extensive hydrophobic interactions with L4, P13 and 155 V16. The residues in ECL2 of GALR1 involved in binding galanin have bulkier aromatic side 156 chains than that in GALR2 (Fig. 2F). Mutations of the equivalent residues W188 ${ }^{\mathrm{ECL} 2}$ and $157 \mathrm{H} 176^{\mathrm{ECL2}}$ in GALR1 and GALR2 respectively had distinct effect on galanin potency (Fig. 2 G 158 and $\boldsymbol{H}$ ), suggesting that ECL2 in GALR1 and GALR2 differently contribute to galanin binding. 159 An endogenous peptide spexin has A7M and G8L mutations in galanin and specifically 160 activates GALR2 and GALR3 (SI Appendix, Fig. S4A). Structure of the spexin-bound 161 GALR2-Gq complex reveals that L8 in spexin likely clashes with the bulkier residue $162 W^{W} 188^{E C L 2}$ in GALR1 (SI Appendix, Fig. S4C), accounting for the specific binding of spexin 163 for GALR2 and GALR3 (16). Owing to these conformational differences, R184 ${ }^{5.35}$ in GALR2 164 but not $\mathrm{K} 197^{5.35}$ in GALR1 makes hydrogen bonds with the backbone of galanin (Fig. 2F). 
165 As a result, $\mathrm{R} 184^{5.35} \mathrm{~A}$ mutation reduces galanin potency by about 10 -fold, while $\mathrm{K} 197^{5.35} \mathrm{~A}$

166 mutation shows little effect (Figs. $2 \boldsymbol{G}$ and $\boldsymbol{H}$ ). Taken together, these results suggest that 167 mechanisms of galanin recognition by GALR1 and GALR2 are not identical, which allows the 168 development of selective ligands targeting a specific subtype.

\section{Activation mechanisms of GALR1 and GALR2}

171 The inactive structures of GALR1 and GALR2 predicted by Alphafold may represent

172 ligand-free structure, in which TM helices loosely pack against each other to allow the 173 access of galanin (32) (Fig. 3A). Upon galanin binding, the orthosteric site undergoes 174 significant conformational change, as indicated by the inward displacement of the 175 extracellular portions of TM2 and TM6 and the outward shift of the TM1 and TM7 (Figs. $3 \boldsymbol{A}$ 176 and $B$ ). These conformation changes account for the outward motion of TM6 and inward 177 motion of TM7 in the intracellular side (Fig. 3C). The conformational changes of the toggle 178 switch $\mathrm{W}^{6.48}$ and $\mathrm{P}^{5.50} \mathrm{I} / \mathrm{V}^{3.40} \mathrm{~F}^{6.44}$ motif are common features of the class A GPCR activation. 179 In contrast to the most class A GPCRs, where the orthosteric sites are in close proximity to 180 the toggle switch, galanin binding site is distant from it. Hydrophobic interactions between 181 F275 in ECL3 of GALR1 and, L10 and L11 in galanin result in the downward shift of F275, 182 which propagates to the downward movement of $\mathrm{W}_{26} 60^{6.48}$ via I266 6.54 and H263 ${ }^{6.51}$ (Fig. 3B). 183 The downward shift of $W^{6.48}$ is associated with the conformational change of the $184 \mathrm{P}^{5.50} \mathrm{I} / \mathrm{V}^{3.40} \mathrm{~F}^{6.44}$ motif, which allosterically disrupts the conserved ionic lock between $\mathrm{R} 133^{3.50}$ 185 and D132 3.49 , leading to the outward displacement of TM6 (Fig. 3C). Inward displacement of 186 TM7 in the intracellular side is observed in the active state of many other class A GPCRs, as 187 indicated by the conformational change of NPXXY motif, in which Y $303^{7.53}$ forms a 188 water-meditated hydrogen bond with $Y 220^{5.58}$. The inward displacement of TM7 is coupled 189 by the outward shift of R285 $5^{7.35}$ that arises from its interaction with galanin. Although the key 190 residues involved in receptor activation are conserved between GALR1 and GALR2, their 191 conformations vary significantly (Fig. 3D). This is because the hydrophobic interaction 192 between V274 in ECL3 and L11 in galanin exists in GALR1, while this interaction is absent in 
193 GALR2 due to the substitution of V274 in Q263, which leads to the upward shift of F264 ${ }^{\mathrm{ECL} 3}$ 194 as well as the toggle switch $\mathrm{W}^{6.48}$ and PIF motif in GALR2, compared to the equivalent 195 residues in GALR1 (Fig. 3D). To further support the important role of F275 ${ }^{\mathrm{ECL} 3} / \mathrm{F} 264^{\mathrm{ECL} 3}$ in

196 GALR receptors activation, mutation of F275 in GALR1 or F264 in GALR2 reduced galanin 197 potency by almost 100 -fold (Figs. $2 \boldsymbol{G}$ and $\boldsymbol{H}$ ). The conformation differences of residues 198 involved in receptor activation contribute to the structural variation in the cytoplasmic pocket 199 of GALR1 and GALR2 and may play a role in G protein selectivity.

$201 \mathrm{Zn}^{2+}$ is a negative allosteric modulator (NAM) of GALR1

202 Previous studies have reported that $\mathrm{Zn}^{2+}$ can inhibit galanin binding to the receptors (18). To 203 further investigate the functional role of zinc ion in galanin receptors signaling, we used the 204 NanoBiT complementation-based assay to assess the effect of $\mathrm{Zn}^{2+}$ on activation of 205 receptors by galanin in living cells. Mini-G proteins were used in the NanoBiT assay 206 throughout this study, since they preserve appropriate coupling specificity, and can be 207 recruited to the active GPCRs without further dissociation, which increases the 208 signal-to-noise ratio in this assay. As expected, $\mathrm{Zn}^{2+}$ diminished the effect of $1 \mu \mathrm{M}$ galanin on 209 GALR1 activation in a concentration dependent manner with an IC50 value of $47.2 \mu \mathrm{M}$ (Fig. $2104 A$ ). The diminished effect of $\mathrm{Zn}^{2+}$ is saturable, or has a "ceiling" level. In contrast, the 211 diminished effect was observed in GALR2 when the concentration of $\mathrm{Zn}^{2+}$ reached millimolar 212 range that is above the physiological concentration, indicating that $\mathrm{Zn}^{2+}$ had little effect on 213 galanin-induced GALR2 activation. Our structures show that galanin receptors are enriched 214 with histidine residues that may coordinate $\mathrm{Zn}^{2+}$ underneath the orthosteric binding pocket 215 (Fig. 4B). Comparison of primary sequences of GALR1 and GALR2 from different species 216 revealed that $\mathrm{H} 267^{6.55}$ but not nearby histidine residues in GALR1 is mutated to Isoleucine in 217 GALR2 (SI Appendix, Fig. S5A). As expected, the zinc effect was significantly abrogated in 218 GALR1 when $\mathrm{H} 267^{6.55}$ was mutated, while $\mathrm{H} 112^{3.29} \mathrm{~A}, \mathrm{H} 263^{6.51} \mathrm{~F}$ or $\mathrm{H} 264^{6.52} \mathrm{~F}$ mutation had 219 little influence (Fig. 4A and SI Appendix, Fig. S5B). All these mutants of GALR1 can be 220 activated by galanin, although the potency and efficacy of galanin for these mutants vary (SI 
221 Appendix, Fig. S5C). We further tested the effect of $\mathrm{Zn}^{2+}$ on the concentration response

222 curve of galanin. $\mathrm{Zn}^{2+}$ produced the concentration-dependent and saturable rightward shifts

223 in the potency of galanin, and decreased the galanin maximum response as well (Fig. 4C).

224 By contrast, $\mathrm{Zn}^{2+}$ had little effect on the galanin concentration-response curve of $\mathrm{H} 267 \mathrm{~A}$

225 mutant of GALR1 (Fig. 4D). H267 is located in the TM6 right below the galanin binding site.

226 The extracellular part of TM6 near H267 moves inwards upon galanin binding, which leads

227 to the receptor activation. As a result, when coordinated by $\mathrm{H} 267$ and other nearby residues,

$228 \mathrm{Zn}^{2+}$ likely rigidifies the extracellular part of TM6 and restricts its conformational change,

229 attenuating galanin-induced receptor activation. The exact coordination pattern of $\mathrm{Zn}^{2+}$

230 awaits further investigation. Nevertheless, these results indicate that $\mathrm{Zn}^{2+}$ is a NAM of

231 GALR1.

232

\section{Structural determinants of $\mathrm{Gi} / \mathrm{o}$ and $\mathrm{Gq} / 11$ selectivity}

234 A notable difference between structures of the GALR1-Go complex and the GALR2-Gq 235 complex is the relative orientation of $\mathrm{Go}$ and $\mathrm{Gq}$ to the receptors (Figs. $5 \boldsymbol{A}$ and $\boldsymbol{B}$ ). When 236 aligning the receptors, the $a 5$ of Gao is rotated around the "wavy hook" of a5 by about $14^{\circ}$ 237 toward TM5, compared with Gaq. This orientation difference was also observed in the 238 structures of $M 1$ and M2 muscarinic receptors (M1R and M2R) bound to G11 and Go 239 respectively (33). In addition, because of the different interaction interface of the receptor 240 and G protein, the flexibility of intracellular loops (ICL) in GALR1 and GALR2 differs (Figs.

$2415 \boldsymbol{A})$. For instance, ICL1 is ordered in GALR2 owing to the hydrogen bond interaction 242 between D312 in G $\beta$ and the main chain carbonyl group of G53 in ICL1, whereas it is flexible

243 in GALR1 due to the absence of this interaction (SI Appendix, Fig. S6A). The ICL2 of most

$244 \mathrm{Gi}$ /o-coupled receptors forms an alpha helical structure, where hydrophobic residues at 245 position 34.51 engage weak hydrophobic interactions with the hydrophobic pocket of Gai/o

246 formed by V34 from the aN- $\beta 1$ loop, L195 from the $\beta 2-\beta 3$ loop and I343 and F336 from a5

247 (SI Appendix, Fig. S6B). However, the ICL2 of GALR1 is disordered because of the 248 substitution of the hydrophobic residue $L 1313^{4.51}$ in arginine and the absence of hydrophobic 
249 interaction between the ICL2 of GALR1 and Gi (Figs. 5 C and G). By contrast, L131 $1^{34.51}$ in

250 the ICL2 of GALR2 is buried deep in the hydrophobic pocket of Gaq formed by L34 from the $251 \alpha \mathrm{N}-\beta 1$ loop, V79 from the $\beta 2-\beta 3$ loop, and F228, K232 and I235 from $\alpha 5$, and engages 252 strong hydrophobic interactions. In addition, P130 34.50 at the junction of ICL1 and TM3 is 253 stabilized through hydrophobic interactions with 1235 and K232 in a5 of Gaq (Fig. 5D). 254 Substitution of $\mathrm{L} 131^{34.51}$ or $\mathrm{P} 130^{34.50}$ in GALR2 in the equivalent residues in GALR1 impaired

255 the ability of GALR2 to couple Gq (SI Appendix, Fig. S7A), accounting for the inability of 256 GALR1 to couple Gq. However, substitutions of $S 140^{34.50}, \mathrm{R} 141^{34.51}$ and S149.49 in ICL2 of 257 GALR1 with the equivalent residues in GALR2 have little effect on coupling efficiency 258 between GALR1 and Gai (SI Appendix, Fig. S7B), suggesting that ICL2 in GALR1 is not 259 involved in Gi coupling. Remarkably, GALR1 acquires the ability to couple Gq, as indicated 260 by the NanoBiT assay as well as the IP1 assay (Fig. 5 H and SI Appendix, Fig. S7C), when 261 residues in ICL2 of GALR1 are substituted with that of GALR2, further supporting the 262 important role of ICL2 in Gq coupling.

263

264 To understand the structural mechanism of the inability of GALR2 to couple Gi, we 265 compared the interaction details between the GALR1-Gi and the GALR2-Gq complexes 266 and mainly focused on residues of GALR1 involved in Go coupling that are not conserved in

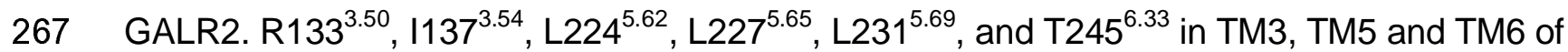

268 GALR1 engage extensive hydrophobic interactions with the extreme C-terminal part of a5 in 269 Go (Fig. 5E). Most of these residues are conserved in GALR2 (Fig. 5F). Mutations of 270 conserved residues in GALR1 and GALR2 impair the recruitment of Gi and Gq, respectively 271 (SI Appendix, Figs. S7D-7G). Nevertheless, a notable difference between GALR1 and 272 GARL2 are in ICL3. S238 ${ }^{\mathrm{ICL3}}$ and S235 ${ }^{\mathrm{ICL} 3}$ in ICL3 of GALR1 make hydrogen bonds with $273 \mathrm{D} 341$ in Gao, and $\mathrm{K} 237^{\mathrm{ICL} 3}$ engages electrostatic interactions with residues in the GTPase 274 domain of Gao. All of three residues are mutated in GALR2, leading to loss of these 275 interactions (Fig. $5 \boldsymbol{E}$ and $\boldsymbol{F}$ ). Mutations of $\mathrm{S} 235^{\mathrm{ICL3}}$ and $\mathrm{K} 237^{\mathrm{ICL} 3}$ in GALR1 have modest 276 effect on galanin potency, but dramatically reduced the maximum responses (SI Appendix, 
277 Figs. S7D and S7E). Remarkably, GALR2 acquired the ability to bind Go, when ICL3 278 (214-225) of GALR2 including the three residues were replaced by the equivalent residues 279 in GALR1 (Fig. 5I). Taken together, our data suggest that ICL2 in GALR2 and ICL3 in

280 GALR1 are critical for determining the Gq and Go selectivity, respectively.

281 Structural determinants of Gs and Gq selectivity

282 Although it has been shown that interactions between the hydrophobic residue at position

28334.51 of ICL2 and the hydrophobic pocket of $\mathrm{Ga}$ are essential for the efficient coupling of $\mathrm{Gq}$ 284 and $\mathrm{Gs}$ (34), it remains unclear how $\mathrm{Gq}$ and $\mathrm{Gs}$ are selectively recognized. Comparison of 285 structures of D1 dopamine receptor (D1R)-Gs and GALR2-Gq revealed key structural 286 elements in the receptors that determine $\mathrm{Gq}$ and $\mathrm{Gs}$ selectivity. In the GALR2-Gq complex, 287 the conformation of ICL2 is stabilized by salt bridge interactions between $R^{34.57}\left(M^{34.57}\right.$ in $288 \mathrm{D} 1 \mathrm{R})$ and $\mathrm{D}^{3.49}$ of the DRY motif as well as a hydrogen bond between $\mathrm{R}^{34.57}$ and $\mathrm{Y}(-4)$ in $289 \mathrm{Gaq}$, while ICL2 of D1R is stabilized by a hydrogen bond between $\mathrm{Y}^{34.53}$ ( $\mathrm{S}^{34.53}$ in GALR2) 290 and $D^{3.49}$, and a potential water-meditated hydrogen bond between $Y^{3.49}$ and $Y(-4)$ in Gas 291 (Fig. 6A). Notably, $\mathrm{Y}^{34.53} \mathrm{M} / \mathrm{V}^{34.57}$ are prevalent in Gs-coupled receptors, while $\mathrm{R}^{34.57}$ is 292 enriched in Gq-couple receptors (Fig. 6D). Mutations of YM in D1R and RS in GALR2 293 significantly reduced the potency of dopamine and galanin, respectively (Figs. $6 \boldsymbol{E}$ and $\boldsymbol{F}$ ). 294 Moreover, N(-3) (-1 indicates the last residue of $\mathrm{Ga}$ ) in Gaq is inserted into a hydrophobic 295 pocket formed by $\mathrm{N}^{2.40}, \mathrm{~F}^{8.50}$ and other nearby residues, whereas $\mathrm{E}(-3)$ flips outside this 296 pocket, probably due to its longer side chain and negative-charge nature. Remarkably, when $297 E(-3)$ in Gas but not the nearby residues $L(-1)$ and $Q(-5)$ was substituted with the equivalent 298 residues in Gaq, the coupling efficiency between GALR2 and Gs was significantly increased

299 (Fig. 6G). These different interaction modes of GALR2-Gq and D1R-Gs account for the 300 movement of a5 in Gs toward TM6 and the outward movement of TM6 in D1R, compared to 301 that in GALR2 (Fig. 6B), explaining that most Gs-coupled receptors display a larger TM6 302 movement in the active state than Gq-coupled receptors. As a result of these conformational 303 changes, Gs is closer to TM5 than Gq, highlighting the important role of TM5 in determining 304 Gs selectivity. Indeed, TM5 in most Gs-coupled receptors have a C-terminal helical 
305 extension, and previous studies have shown that the $A / V^{5.65} Q^{5.68} \Phi^{5.69}(\Phi$ represents 306 hydrophobic residues) motif in TM5 is prevalent in receptors that exclusively couple to Gs 307 and is critical for Gs coupling in D1R (35) (Companion paper). Residues at position 5.65 in

308 Gs-coupled receptors prefer hydrophobic residues with small side chains such as alanine 309 and valine because of its close distance from the hydrophobic pocket formed by $L(-1), L(-2)$, 310 and $L(-7)$ in Gas (Fig. 6C). Mutation of $A^{5.65}$ in leucine would cause a clash with this pocket, 311 and impaired the Gs coupling (Companion paper). In contrast, leucine is dominant at 312 position 5.65 in Gq-coupled receptors, due to its long distance from the hydrophobic pocket 313 formed by $V(-1), L(-2)$, and $L(-7)$ in Gaq (Fig. 6C). $L^{5.65} A$ mutation in GALR2 weakens the 314 interaction with this hydrophobic pocket and thus significantly decreased galanin potency 315 (Fig. 6F). However, it is noteworthy that Gs- and Gq-coupled receptors show sequence 316 preference at some positions of ICL2 and TM5, but also accommodate various residues at 317 these positions (Fig. 6D), partly because of diverse receptor-G protein interfaces and 318 promiscuous coupling of some GPCRs.

\section{Discussion}

321 Here, we report cryo-EM structures of the GALR1-Go and GALR2-Gq complex using the 322 GPCR-G protein fusion strategy. The structures revealed distinct mechanisms of galanin 323 recognition and receptor activation for GALR1 and GALR2, which contribute to structural 324 variation in the cytoplasmic pocket of the receptors and may play an important role in 325 determining the $\mathrm{G}$ protein selectivity. Moreover, we showed that $\mathrm{Zn}^{2+}$ is a negative allosteric 326 modulator of GALR1 but not GALR2.

$328 \mathrm{Zn}^{2+}$, known as a neuromodulator, is widely distributed in the central nervous system (CNS), 329 particularly enriched in the synaptic vesicles of glutamatergic neurons $(36,37)$. It is released 330 to the synaptic cleft upon membrane depolarization, and modulates functions of ion 331 channels and receptors on the pre- or post-synaptic membrane. It has been shown that zinc 332 inhibits ionotropic glutamate AMPA and NMAR receptors, fine-tuning synaptic transmission 333 in the brain $(38,39)$. GALR1 is expressed on both glutamatergic and GABAergic 
334 postsynaptic neurons. The spatial colocalization of zinc and GALR1 makes it possible for 335 zinc to regulate the function of GALR1. Moreover, the IC50 of zinc on GALR1 activation is $33647.2 \mu \mathrm{M}$, which is in the range of the physiological concentration of zinc (10 $\mathrm{nM}$ to $100 \mu \mathrm{M})$ 337 (40). Previous studies have also shown that zinc regulates endogenous ligand binding at 338 several GPCRs including $\beta 2$ adrenergic receptors ( $\beta 2 A R$ ) (41), melanocortin receptors (42) 339 and platelet-activating factor receptor (43). In this study, we showed that zinc attenuated

340 GALR1 activation by galanin possibly through restricting the conformational change of TM6 341 that leads to receptor activation. Further studies are required to address whether zinc 342 modulates a large number of GPCRs in the CNS and fine-tunes GPCR signaling, as does 343 sodium (44).

345 Combining published structures of the GPCR-Gi complexes, we can roughly divide the class 346 A Gi-coupled receptors into three classes based on the interaction features between ICL2 347 and G proteins: (i) receptors that exclusively couple to Gi and have a charge residue at the 348 position 34.51 of ICL2 such as GALR1 and sphingosine-1-phosphate receptors (S1PR) (45);

349 (ii) receptors that exclusively couple to $\mathrm{Gi}$ and have a hydrophobic residue at the position 35034.51 such as D3 dopamine receptor, M2 muscarinic receptor (M2R) and $\mu$ opioid receptor 351 (28, 33, 46); (iii) receptors that promiscuously couple to Gi and have a large hydrophobic 352 residue at position 34.51 such as the neurotensin receptor 1 (NTSR1), $\beta 2 A R$, and the 353 cholecystokinin A receptor $(47,48)$. In the first class, when bound to the receptor, ICL2 is 354 disordered, or forms a random coil structure. Since there is no hydrophobic interaction 355 between 34.51 and Gai, ICL2 in receptors of this class plays a distinct role in determining Gi 356 coupling efficiency (45) (SI Appendix, Fig. S8A). In GALR1, ICL2 is not involved in Gi 357 coupling, whereas in S1PR, ICL2 is involved in hydrophilic interactions with Gi, and is 358 important for Gi coupling; In the second class, ICL2 forms an alpha helical structure, and the 359 residue 34.51 of ICL2 is located outside and distant from the hydrophobic pocket of Gai 360 formed by residues from the $\alpha \mathrm{N}-\beta 1$ loop, the $\beta 2-\beta 3$ loop and $\alpha 5$, and engages weak 361 hydrophobic interactions (SI Appendix, Figs. S8B and S8C). Mutation of this residue had 
362 little effect on the Gi coupling or GDP release from Gi/o $(49,50)$; In the third class, similar to

363 Gs- and Gq-coupled receptors, the residue 34.51 is located close to the middle of the

364 hydrophobic pocket of Gai and engages strong hydrophobic interaction (SI Appendix, Figs.

365 S8E and S8F). In addition, some receptors in this class such as NTSR1 have the other

366 conformation, where the residue 34.51 is located outside the hydrophobic pocket (SI

367 Appendix, Fig. S8D). Previous studies have shown that $F^{34.51}$ A mutant of $\beta 2 A R$ failed to

368 activate $\mathrm{Gi}(50)$, suggesting that the hydrophobic interaction between ICL2 and Gi is very

369 important for Gi coupling in the third class. Owing to the absence of or weak interaction

370 between Gai and ICL2 in receptors that exclusively couple Gi, the cytoplasmic end of TM5

371 and TM6, and ICL3 have strong interactions with Gai, and are critical for determining Gi

372 selectivity (SI Appendix, Fig. S8).

374 It has been recognized that the distal part of a5 in Ga plays a key role in determining $\mathrm{G}$ 375 protein selectivity (51-53). We further identified a residue pair $N / E(-3)$ in $a 5$ of $G q / G s$ that 376 contributes to structural differences and selective interactions between the D1R-Gs and 377 GALR2-Gq. Substitution of this residue in Gs can promote coupling of GALR2 to noncognate 378 Gs. Moreover, we revealed several signature residues in ICL2 and TM5 that dominate in Gs379 and Gq-coupled receptors. Thus, our results provide novel insights into the molecular 380 mechanisms of G protein selectivity by class A GPCRs. 


\section{Figure legends:}

A

Galanin: GW TLNSAG Y LL GPHAVGNHRSF S KN GLTS

Galanin receptor (GALR)
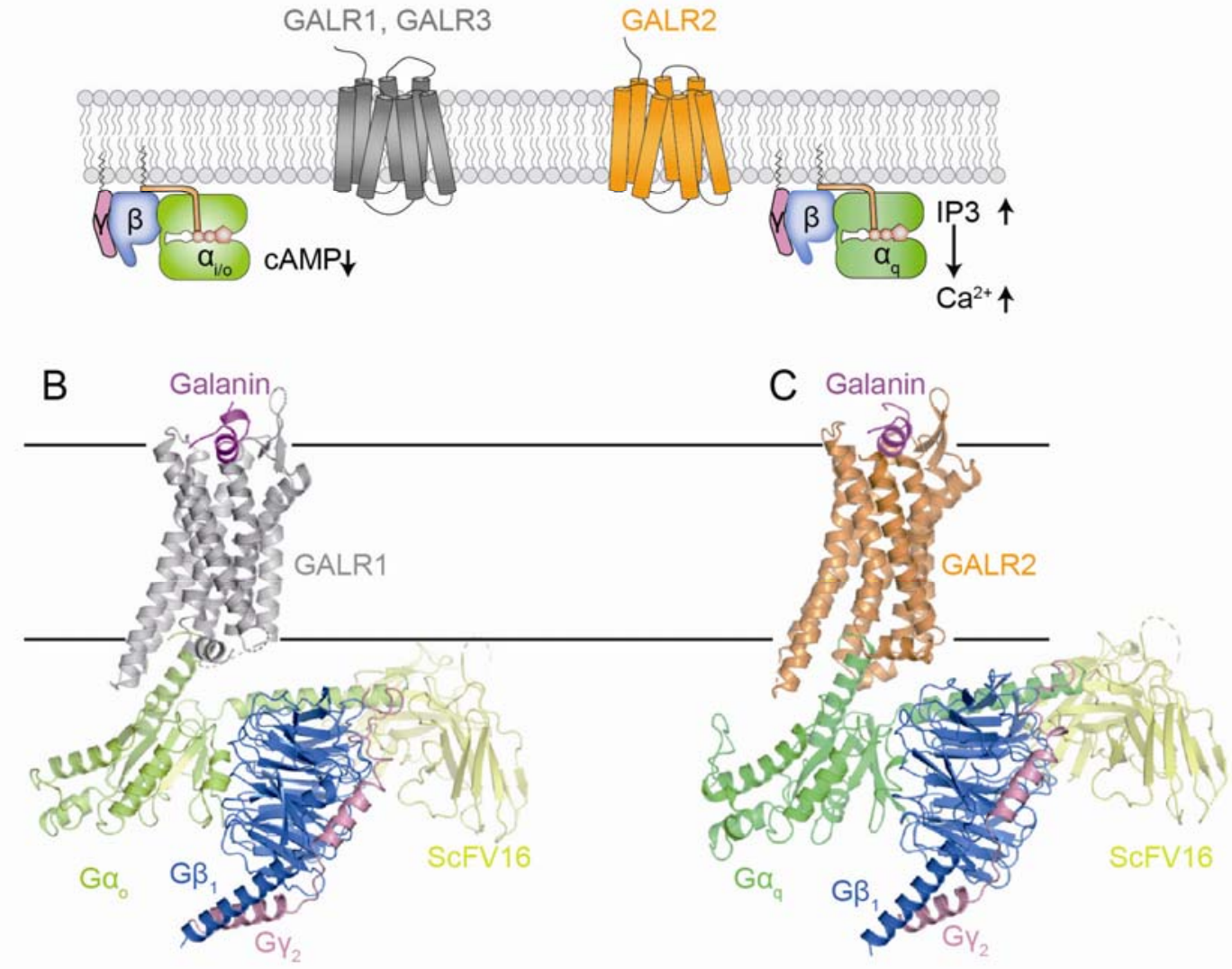

386 Fig. 1. Overall structures of galanin-bound GALR1-miniGo-scFv16 and

387 GALR2-miniGq-scFv16 complexes.

388 (A) Schematic representation of GALR receptors signaling. GALR1 and GALR3 primarily 389 couple to Gi/o, while GALR2 mainly signals through Gq.

390 (B) Cryo-EM structures of GALR1-miniGo-scFv16.

391 (C) Cryo-EM structures of GALR2-miniGq-scFv16. 
A
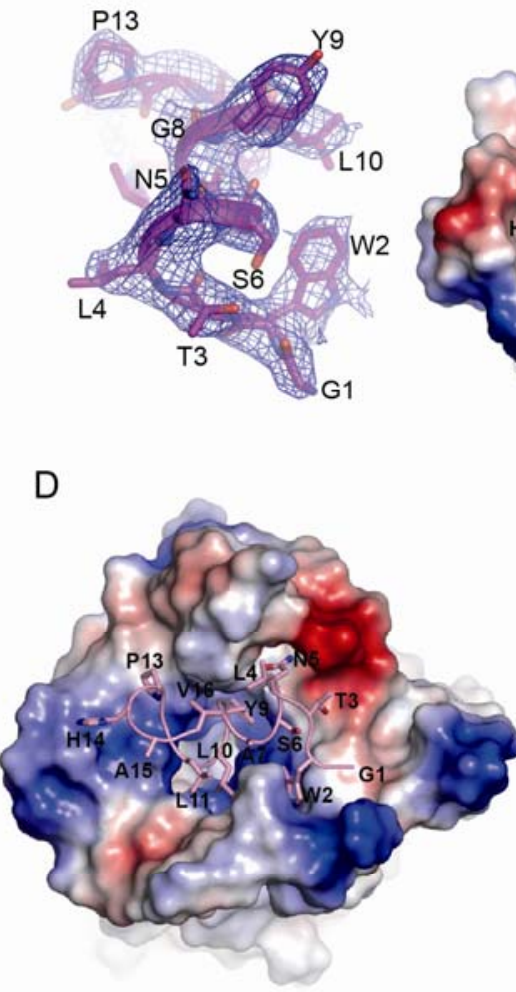

B

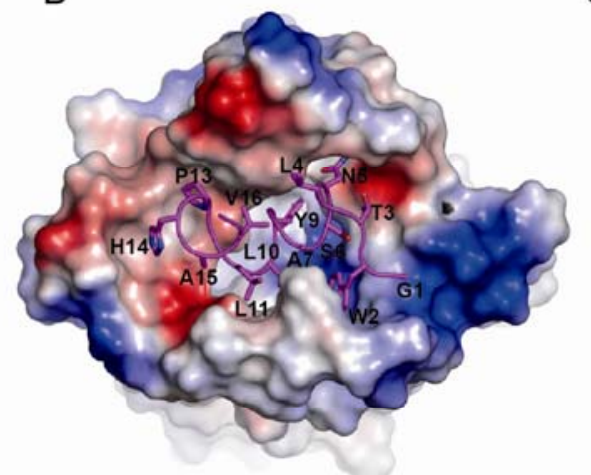

$\mathrm{E}$
GALR1

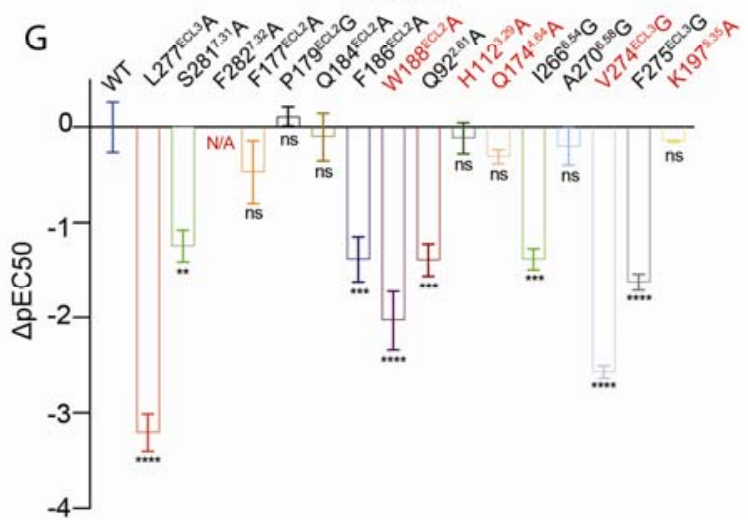

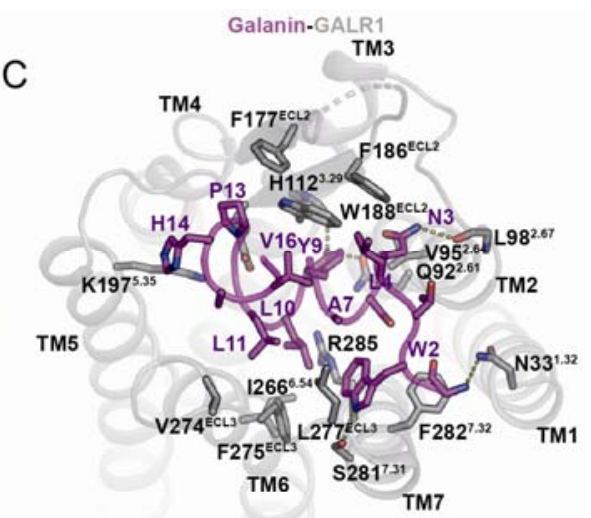

$\mathrm{F}$

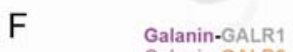

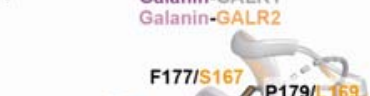
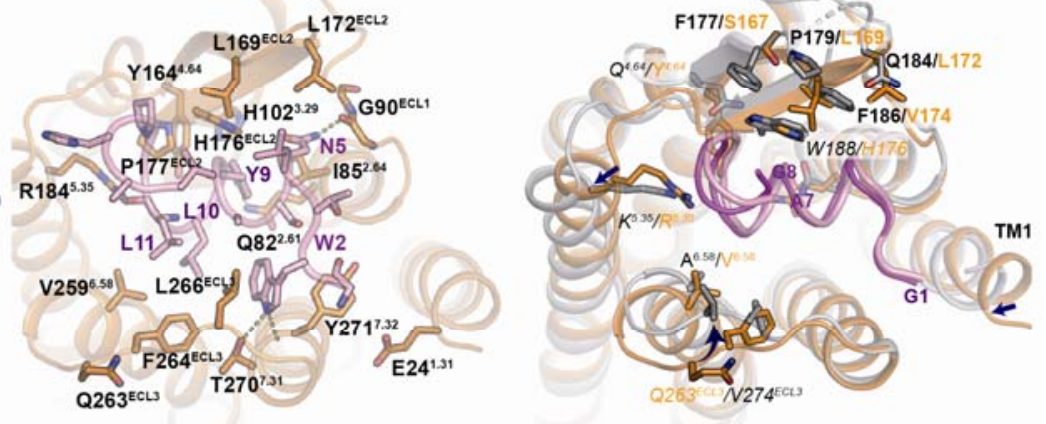

GALR2

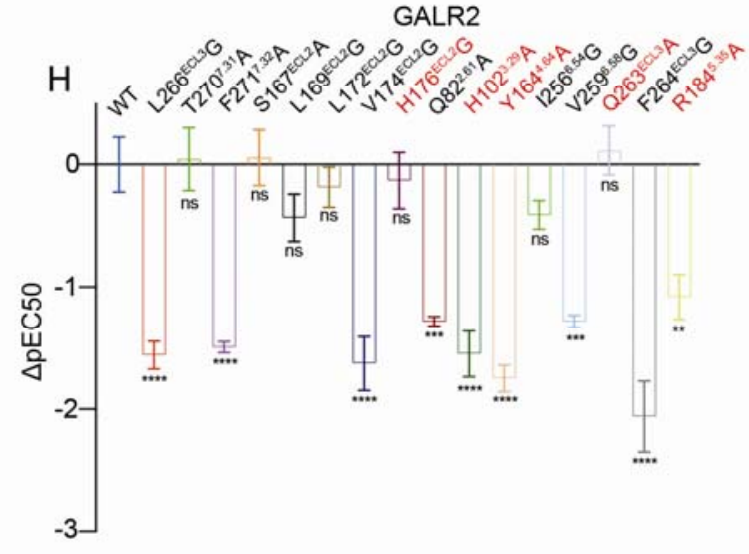

(B) Electrostatic potential surface of GALR1 and ribbon representation of galanin (magenta) viewed from the extracellular side. Colors from red to blue represent negatively to positively charged regions.

(C) Detailed interaction between GALR1 and galanin.

(D) Electrostatic potential of the GALR2-galanin interface is distinct from that of the 400 GALR1-galanin interface.

401 (E) Detailed interaction between GALR2 and galanin. 
403 equivalent residues in GALR1 and GALR2 that play distinct roles in galanin binding are 404 shown. Arrows indicate the conformational changes.

$405(\mathrm{G})$ and $(\mathrm{H})$ The effects of mutations in GALR1 and GALR2 on galanin potency as measured 406 by the cAMP inhibition assay and the IP1 accumulation assay respectively. The equivalent 407 residues that play distinct roles in GALR signaling are colored red. Data represent mean \pm 408 SEM of triplicate measurements in three independent experiments. Significance was 409 analyzed using one-way ANOVA, ${ }^{* * *} \mathrm{P}<0.0001,{ }^{* * *} \mathrm{P}<0.001,{ }^{* *} \mathrm{P}<0.01$. 

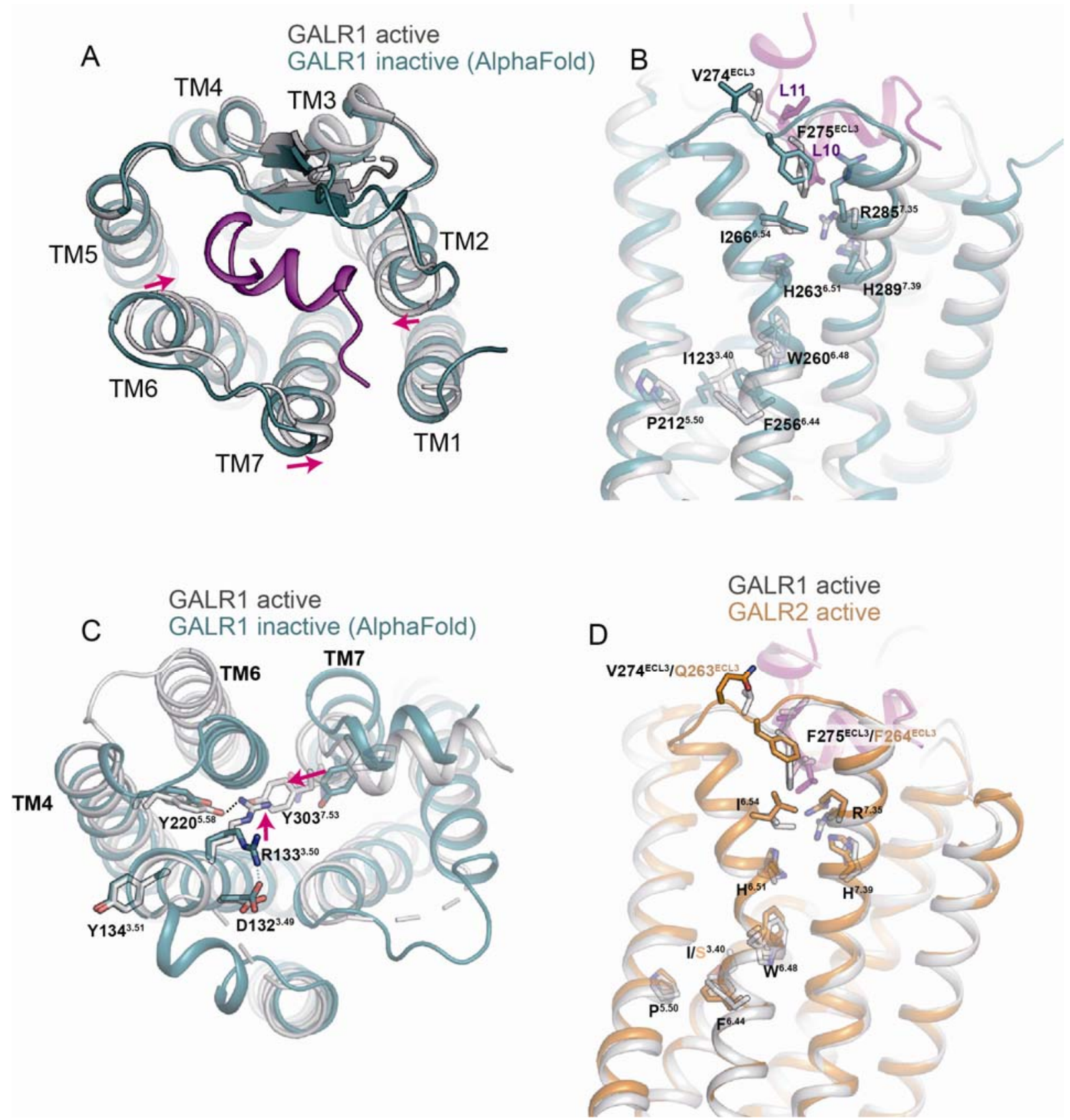

Fig. 3. Mechanisms of GALR1 and GALR2 activation.

413 (A) Structural overlay of GALR1 in the active and inactive state (predicted by AlphaFold).

414 (B) Conformational changes of the $\mathrm{P}^{5.50} \mathrm{~J}^{3.40} \mathrm{~F}^{6.44}$ motif upon receptor activation.

415 (C) Conformational changes of residues in the cytoplasmic pocket including the $416 \quad D^{3.49} R^{3.50} Y^{3.51}$ and the NPY motif upon receptor activation.

417 (D) Structural overlay of the active state of GALR1 and GALR2. 


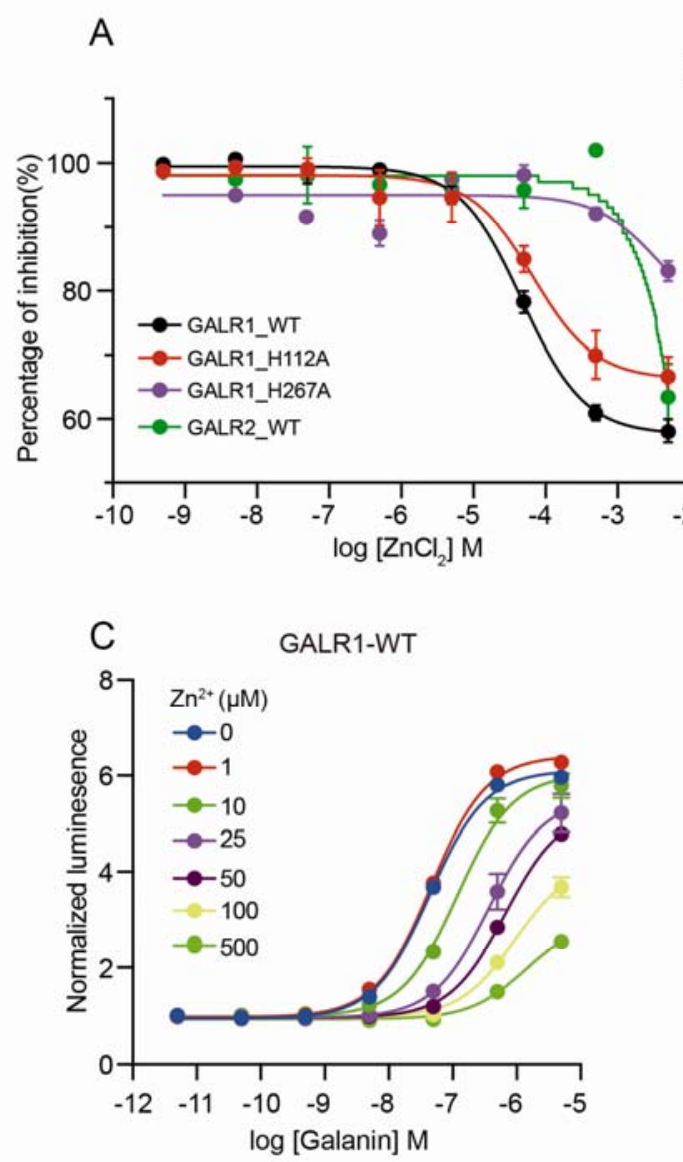

\section{Fig. 4. Zinc is a NAM of GALR1.}

420 (A) The effect of increasing concentration of zinc on receptor activation induced by $1 \mu \mathrm{M}$

421 galanin, as evaluated by the NanoBiT assay, where the small fragment, and the large

422 fragment are fused to the C-terminus of GALR1 and the N-terminus of mini-Go, respectively.

423 The luminescence signals are normalized as percentages of the initial response of GALR1

424 to galanin without zinc treatment.

425 (B) Histidine residues are enriched underneath the galanin binding pocket of GALR1.

426 (C) and (D) The actions of increasing concentration of zinc on the galanin dose-response 427 curve of WT (C) and H267A mutant (D) of GALR1 measured by the NanoBiT assay. The 428 luminescence signals are normalized to the vehicle treatment as fold change. 


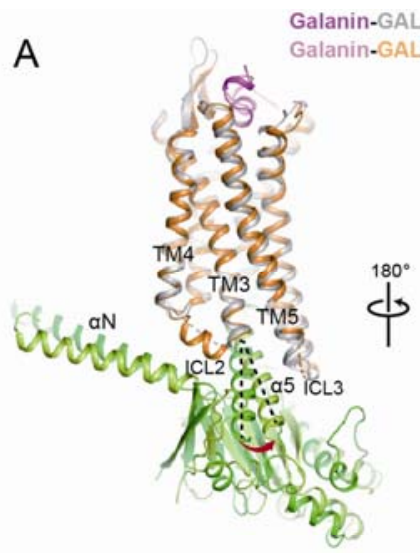

D

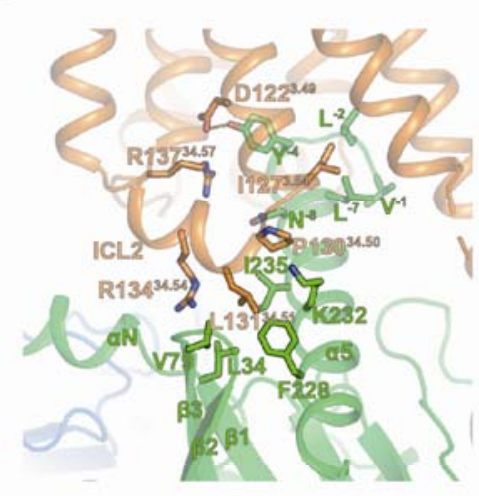

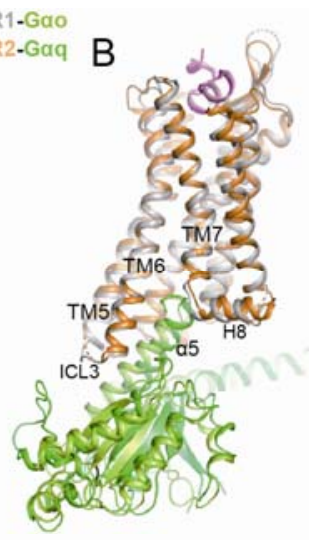

E

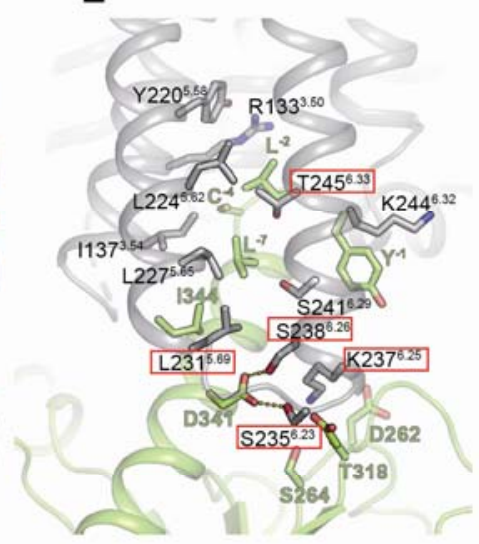

$\mathrm{H}$

ICL2

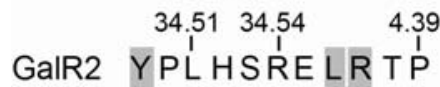

GaIR1 HSRRSSS LR VS

GalR3 HPLRSRALR - -

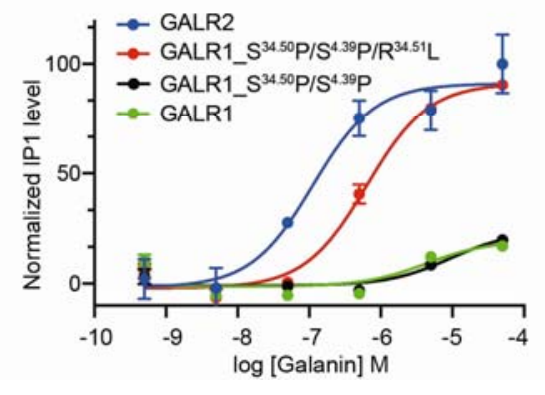

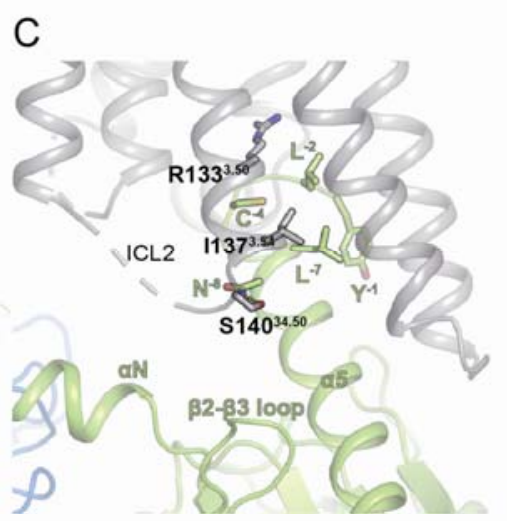

$\mathrm{F}$
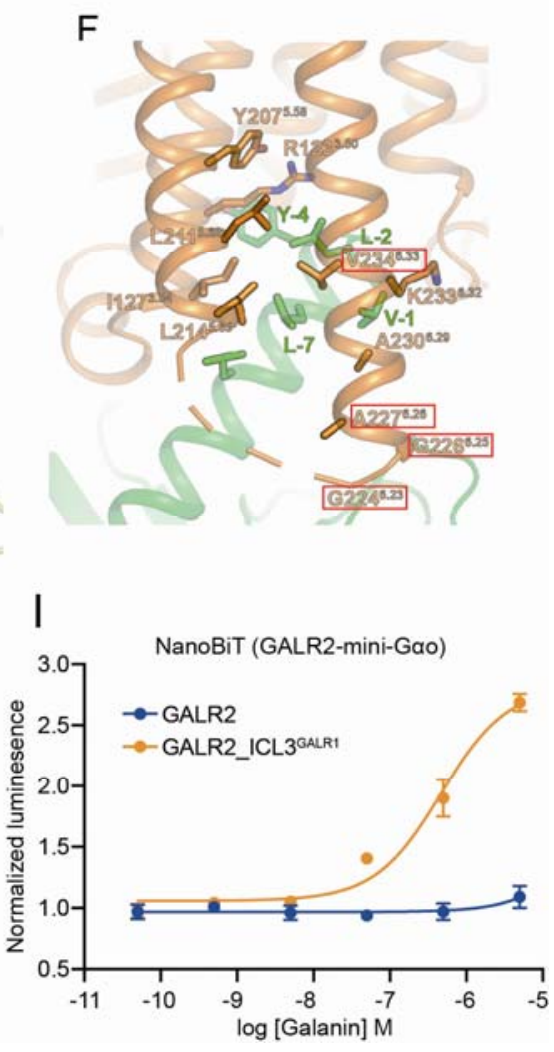

Fig. 5. Mechanisms of Go and Gq selectivity in the GALR receptor family.

(A) and (B) Structural superposition of GALR1-Gao and GALR2-Gaq in two opposite views.

432 Receptors are aligned.

433 (C) Interaction details between ICL2 of GALR1 and Gao.

434 (D) Interaction details between ICL2 of GALR2 and Gaq.

435 (E) Interaction details between TM5 and TM6 of GALR1 and Gao.

436 (F) Interaction details between TM5 and TM6 of GALR2 and Gaq.

437 (G) Sequence alignment of ICL2 from GALR receptor family.

438 (H) The IP1 accumulation assay evaluating the effects of ICL2 substitutions in GALR1 on 439 GALR1-Gq coupling. All mutants are expressed at similar levels as WT.

440 (I) Substitution of ICL3 in GALR2 with that in GALR1 increases coupling efficiency of GALR2 441 and Go. 

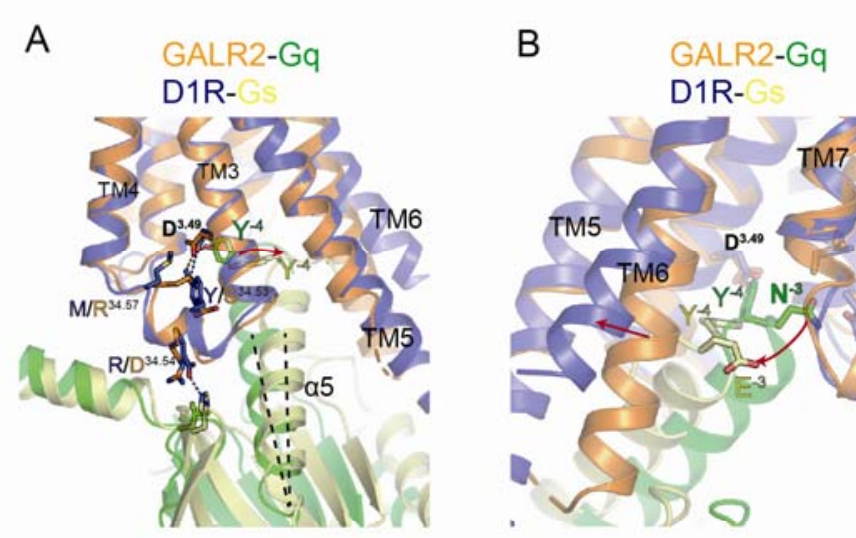

GALR2-Gq
D1R-GS
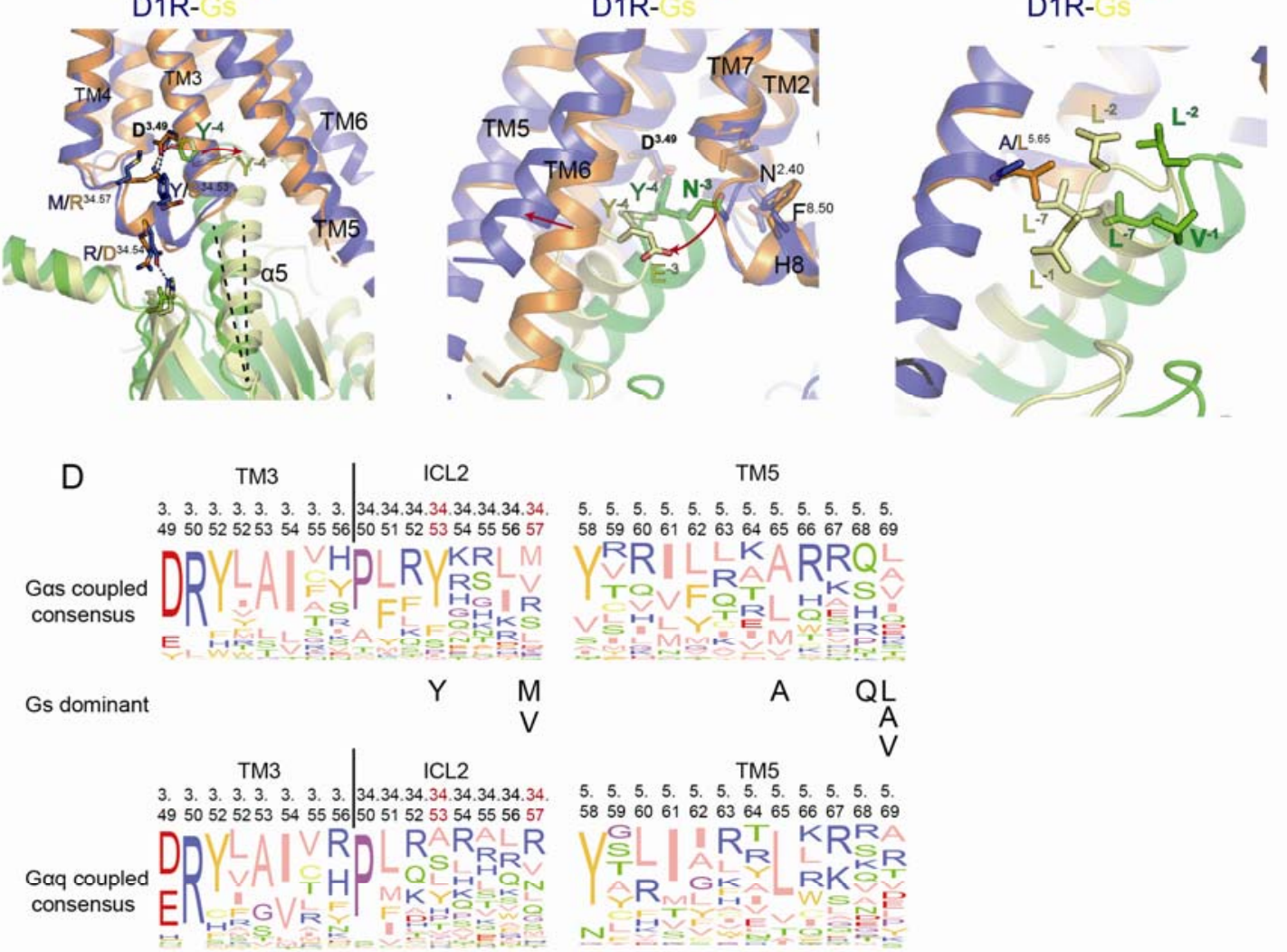

Gq dominant

$R$
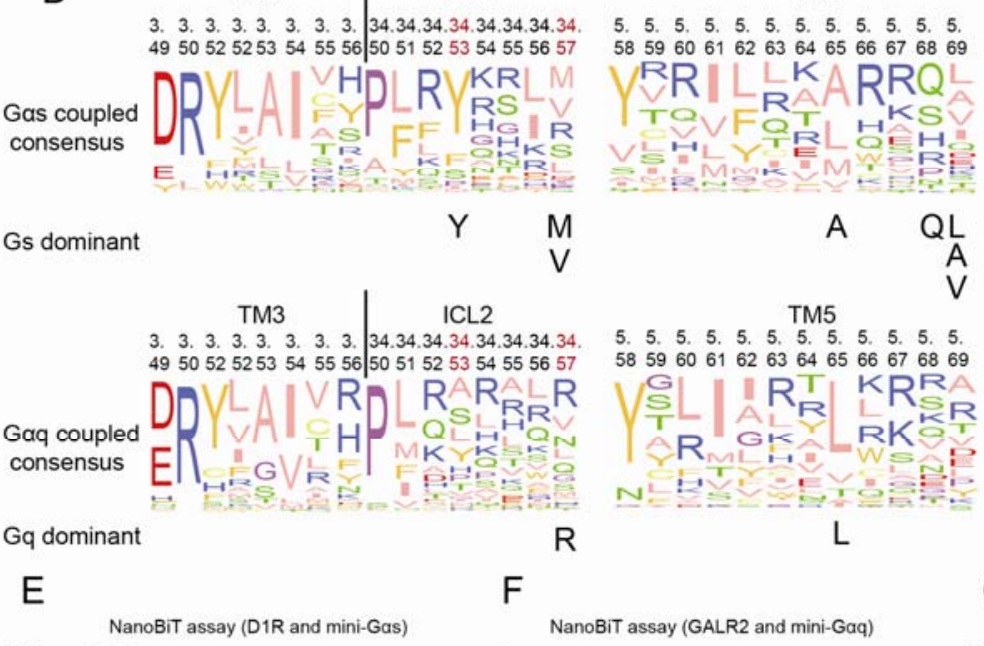

$\mathrm{F}$
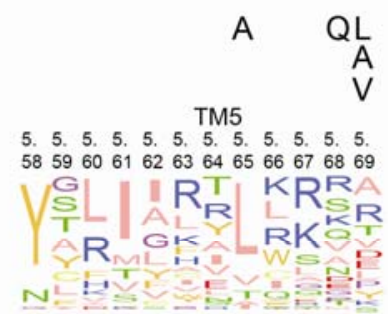

$\mathrm{L}$
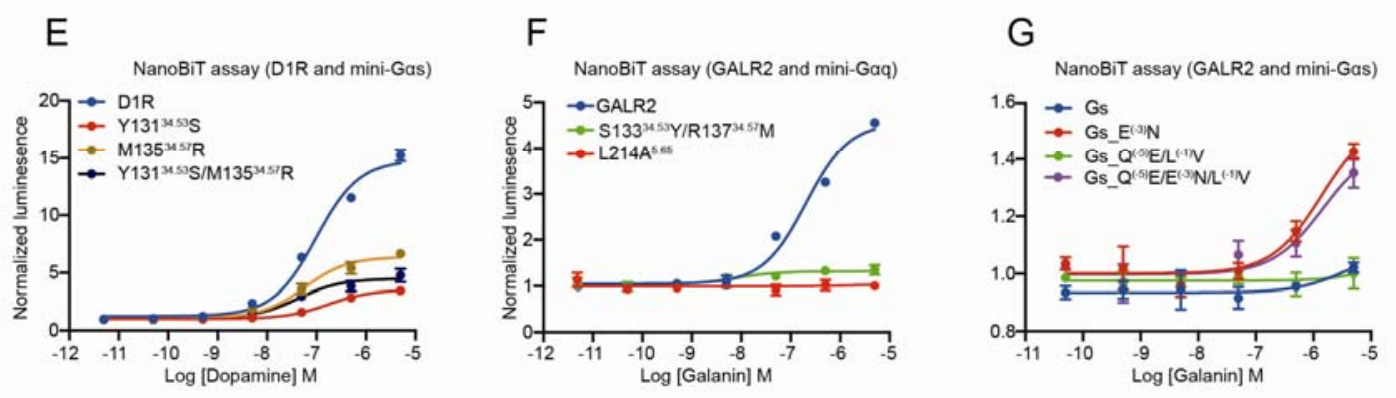

443 Fig. 6. Important structural features in class A GPCRs that determining Gs and Gq 444 selectivity.

445 (A) Structural superposition of the GALR2-Gq and the D1R-Gs complexes. Receptors are 446 aligned.

447 (B) $\mathrm{N}(-3)$ in $\mathrm{Gq}$ is inserted into a hydrophobic pocket formed by TM2, TM7 and H8, while $448 \quad E(-3)$ in Gs flips outside this pocket.

449 (C) $A^{5.65}$ in D1R is close to the hydrophobic pocket formed by $L(-1), L(-2)$ and $L(-7)$ in $G$ s, 450 while $L^{5.65}$ in GALR2 is distant from that in Gq.

451 (D) Sequence alignment of 41 class A Gs-coupled receptors (top) and 44 Gq-coupled 452 receptors (bottom). The dominant residues are indicated below the alignments.

453 (E) Mutations of $\mathrm{Y}^{34.53} \mathrm{M}^{34.57}$ in the ICL2 of D1R reduce D1R-Gs coupling efficiency. 
454 (F) Mutations of $S^{34.53} R^{34.57}$ in the ICL2 of GALR2 almost abolish GALR2 and Gq coupling. 455 (G) The effects of mutations of the "wavy hook" in Gs on coupling efficiency of GALR2-Gas, 456 as evaluated by the NanoBiT assay. 
457 Materials and method

458 Cloning

459 The human GALR1 and GALR2 were cloned into pcDNA3.1(+) vector(Thermo Fisher 460 Scientific) with an N-terminal hemagglutinin (HA) signal sequence and a FLAG epitope tag 461 (DYKDDDDK). An engineered mini-Gao was fused to the C-terminus of GALR1 (1-349) with 462 three copies of $3 \mathrm{C}$ protease sites between them. GALR2 (1-314) was expressed as a fusion 463 protein including two repeats of $3 \mathrm{C}$ protease site and a mini-Gaq sequence in the 464 C-terminus of GALR2. ScFv16 was cloned into the pFastBac vector (Invitrogen) with an 465 N-terminal GP64 signal sequence and a C-terminal 3C protease site, followed by an 466 octa-histidine tag. His6-tagged Gß1 and Gy2 (C68S mutation) were cloned in the pFastBac 467 Dual vector for insect cell expression.

469 Protein expression and purification

470 The plasmid expressing GALR1-mini-Gao or GALR1-mini-Gaq was transiently expressed 471 into Expi293F cells (Thermo Fisher Scientific) using polyethyleneimine (PEI, Polysciences). 472 Cells were lysed in the lysis buffer (20 mM HEPES, pH 7.4) supplemented with protease 473 inhibitor cocktail (Roche) using a glass dounce grinder and centrifuged at 1,000 x $\mathrm{g}$ for 3 474 minutes to remove the nucleus. The membrane fraction was pelleted by centrifugation at $47565,000 \times \mathrm{g}$, at $4{ }^{\circ} \mathrm{C}$ for 1 hour, and homogenized in the solubilization buffer containing $20 \mathrm{mM}$ 476 HEPES pH 7.4, 150 mM NaCl, 1\% Lauryl Maltose Neopentyl Glycol (LMNG), 0.2\% 477 Cholesteryl Hemisuccinate (CHS) and $60 \mathrm{nM}$ galanin peptide (1-30). After centrifugation to 478 remove debris, the supernatant containing solubilized GALRs-mini-Ga was supplemented 479 with $2 \mathrm{mM} \mathrm{CaCl}_{2}$ and loaded onto the M1 anti-FLAG antibody resin. The resin was washed 480 with wash buffer containing $20 \mathrm{mM}$ HEPES, pH 7.4, $300 \mathrm{mM} \mathrm{KCl,} \mathrm{0.01 \%} \mathrm{LMNG,} \mathrm{0.002 \%}$ $481 \mathrm{CHS}, 2 \mathrm{mM} \mathrm{CaCl}_{2}, 10 \mathrm{mM} \mathrm{MgCl}_{2}, 2 \mathrm{mM}$ ATP, $6 \mathrm{nM}$ galanin and eluted with elution buffer 482 containing 20 mM HEPES, pH 7.4, $150 \mathrm{mM} \mathrm{NaCl}, 0.01 \%$ LMNG, 0.002\% CHS, $10 \mathrm{mM}$

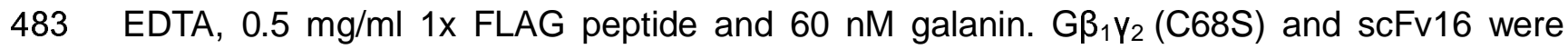
484 expressed and purified as previously described $(21,54)$. 


\section{Complex assembly}

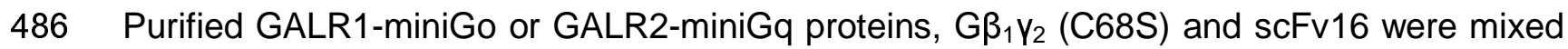
487 with a molar ratio of 1:1.5:2 in $500 \mu$ l of the equilibration buffer (20 mM HEPES, $\mathrm{pH} 7.4,150$ $488 \mathrm{mM} \mathrm{NaCl}, 0.01 \%$ LMNG, 0.002\% CHS, $60 \mathrm{nM}$ galanin, $0.5 \mu \mathrm{M}$ TCEP) supplemented with 1 $489 \mu \mathrm{l}$ PNGaseF and $0.5 \mu \mathrm{l}$ apyrase. The mixture was incubated on ice for 1 hour and further 490 purified on a Superose 6 Increase 10/300 column pre-equilibrated with the equilibration 491 buffer. The peak fractions containing the complex were supplemented with $60 \mu \mathrm{M}$ galanin 492 and concentrated to about $6 \mathrm{mg} / \mathrm{ml}$. For assembly of the GALR1-miniGao/Gß1y2 (C68S) 493 complex with 3C protease site cleaved, similar procedures were performed as above, except 494 that 3C protease was added before purification on a Superose 6 Increase 10/300 column. 495 For assembly of the spexin-bound GALR2 complexes, same procedures were performed, except that galanin was replaced by spexin during the purification process.

\section{Cryo-EM sample preparation and data collection}

499300 mesh holey carbon grids (Quantifoil Au R1.2/1.3) were glow-charged, loaded into a 500 Vitrobot MarkIV instrument chamber (Thermo Fisher Scientific) maintained at $8{ }^{\circ} \mathrm{C}$ and $100 \%$ 501 humidity. $3.0 \mu \mathrm{l}$ of GALRs complex samples was applied onto the grid, blotted for 3.0-4.0 s 502 with a blotting force of 4 before plunge freezing in liquid ethane. Cryo-EM movies were 503 collected on a Titan Krios microscope equipped with a BioQuantum GIF/K3 direct electron 504 detector (Gatan) under accelerating voltage of $300 \mathrm{kV}$ at a nominal magnification of $64,000 \mathrm{x}$. 505 Each movie stack was collected as 32 frames, with total dose of 50 e-/ $/ \AA^{2}$ for $2.56 \mathrm{~s}$.

\section{Cryo-EM Data processing}

508 All movie stacks were collected and processed with MotionCor2 for motion correction (55), 509 with $2 x$ binned to a pixel size of $1.087 \AA$. Contrast Transfer Function (CTF) estimation was 510 performed using patch-based CTF estimation in cryoSPARC_v3 (56). All processed images 511 were then subjected to particle picking using Blob picker in cryoSPARC, followed by particle 512 extraction. For the galanin-bound GALR1-mini-Go complex with 3C protease sites cleaved, 
513 particles from 1,401 micrographs (Dataset A) were subjected to two rounds of 2D

514 classification, generating 248,352 good particles. Ab initio reconstruction and non-uniform 515 refinement were performed to get a reference map for GALR1. For the GALR1-mini-Go

516 fusion protein complex, 886 micrographs (Dataset B) were collected, followed by particle

517 picking using Blob picker and particle extraction. Particles from the two datasets were 518 combined and subjected to two rounds of 2D classification, yielding 2,882,487 good particles.

519 These particles were subjected to global 3D classification in RELION3.1 (57), followed by 520 another round of 3D classification focused on the receptor. 426,045 particles from the best 521 class were run through non-uniform refinement in cryoSPARC, resulting in a final $3.3 \AA$ map.

523 For the galanin-bound GALR2-mini-Gq fusion protein complex, 1,337 micrographs were 524 collected, and processing procedures were performed as above. In brief, two rounds of 2D 525 classification using auto-picked particles resulted in 1,325,739 good particles, which were 526 subjected to two rounds of 3D classification in RELION3.1 using the GALR1-Go complex 527 map as initial model. 578,453 particles from three classes with clear secondary structure 528 features were selected, and subjected to non-uniform refinement in cryoSPARC, resulting in 529 a final $3.29 \AA$ A map. All 3D maps were post-processed with DeepEMhancer (58).

531 For the spexin-bound GALR2-mini-Gq complex, 1,139 movies were collected and processed 532 as above. 1,015,461 good particles were selected from two rounds of 2D classification, and 533 were subjected to heterogeneous refinement and non-uniform refinement in cryoSPARC. 534 The final map is about $3.5 \AA$.

\section{Model building}

537 Homology models for GALR1 and GALR2 were generated using the structure of $\mu$ opioid 538 receptor (PDB: 4DKL) in the SWISS-MODEL server. The homology model of GALR1 and 539 the structure of mini-Gao/Gßy/scFv16 (PDB: 7D77) were fitted into the EM map in Chimera 540 (59). The structure of mini-Gaq/Gßy/scFv16 was extracted from the published structure 
541 (PDB: 6WHA), and docked into the EM map together with the homology model of GALR2.

542 All the models were manually built in COOT (60) and, are subjected to 543 real_space_refinement in Phenix (61) using the reference structure and secondary structure

544 restraints. The statistics for structure refinement are summarized in Table S1.

546 cAMP inhibition assay

547 Chinese hamster ovary $(\mathrm{CHO})$ cells were seeded into six-well plates and cultured overnight 548 until cell confluence reaches $\sim 80 \%$. Plasmids expressing GALR1 or mutants were 549 transfected together with the GloSensor biosensor plasmid following a Lipofectamine cell 550 transfection procedure (Invitrogen). Transfected cells were cultured for 1 day and re-seeded 551 into 96 -well plates by $3 \times 10^{4}$ cells per well. After 8 hours post seeding, the medium was 552 exchanged to CO2-independent medium (Gibco) supplemented with $500 \mu \mathrm{g} / \mathrm{ml}$ of D-luciferin.

553 Cells were stimulated with various concentration gradients of galanin for 5 minutes, and then 554 treated with $1 \mu \mathrm{M}$ forskolin. The bioluminescence signal was constantly measured for 10 555 minutes, and the peak signal was acquired for the inhibitory dose curve fitting and IC50 556 determination using GraphPad Prism 8 software. Significance analysis was performed using 557 one-way analysis of variance method (one-way ANOVA in Prism 8).

\section{IP1 accumulation assay}

560 Gaq-mediated IP1 accumulation was measured using the IP-ONE Gq HTRF Kit from Cisbio. 561 HEK-293T cells were seeded into 6-well plates, and $2 \mu \mathrm{g}$ of GALR2 or mutant plasmids were 562 transfected using PEI. After 2 days post-transfection, cells were suspended, washed one 563 time with DPBS (Gibco), resuspended into HBSS buffer (Beyotine) and seeded into 384-well 564 plates(Greiner) with 7000 cells per well. Transfected cells were stimulated with various 565 concentration gradients of galanin for 1 hour and subjected to IP1 accumulation detection 566 following the assay protocol. Inhibitory dose curve was plotted and IC50 was determined 567 using GraphPad Prism 8 (dose-response-inhibitory, three parameters). Significance was analyzed using One-way ANOVA. 


\section{$570 \quad$ NanoBiT assay}

571 To monitor the interaction between $\mathrm{G}$ proteins and GALR1 or GALR2 upon galanin

572 stimulation, a NanoLuc-based enzyme complementation system called NanoBiT assay (62)

573 was used (Promega). The C-terminus of GALR1 or GALR2 was fused with the small

574 fragment (smBiT), and the large fragment (LgBit) element was fused to the $\mathrm{N}$ terminus of

575 mini-Ga proteins. HEK-293T cells were seeded into 6-well plates and transfected with $1 \mu \mathrm{g}$

576 of GPCR-smBit and $1 \mu \mathrm{g}$ of LgBit-miniGa. After 2 days post transfection, cells were 577 suspended, washed with DPBS for one time and resuspended into the assay buffer 578 containing HBSS supplemented with 0.01\% BSA (SIGMA), 10 mM HEPES (Beyotine) and $57910 \mu \mathrm{M}$ coelenterazine-h (YEASEN). The culture was equilibrated at room temperature (RT) 580 for 2 hours and subjected to stimulation with various concentration gradients of galanin and 581 instant bioluminescence measurement. The bioluminescence signal was acquired in the 582 time point when the signal went into the stationary phase, and the normalized signal (fold 583 change) was fitted to a three-parameter sigmoidal concentration-response curve in Prism 8 584 software.

$\mathrm{Zn}^{2+}$ inhibition assay

587 As zinc produced high background signal in the IP1 accumulation assay and cAMP inhibition 588 assay, the NanoBiT assay was used to measure the effect of $\mathrm{Zn}^{2+}$ effect on GALRs signaling. 589 The same constructs used in the NanoBiT assay were adopted. After 2 days 590 post-transfection, cells were resuspended and washed twice with the assay buffer (20 mM 591 HEPES, pH 7.3 and $150 \mathrm{mM} \mathrm{NaCl}$ ), and resuspended into the assay buffer supplemented 592 with $10 \mu \mathrm{M}$ coelenterazine-h and seeded into 96-well plates. After 30 minutes of incubation 593 at RT, cells were stimulated with various concentration gradients of galanin premixed with a 594 fixed concentration of $\mathrm{ZnCl}_{2}$, or $1 \mu \mathrm{M}$ of galanin pre-mixed with titrated concentration of $595 \mathrm{ZnCl}_{2}$. The bioluminescence signals in the stationary phase were acquired, and were 596 analyzed using three-parameter dose-response-stimulatory or dose-response-inhibitory 
597 fitting methods in Prism 8 software.

598

599

\section{Acknowledgements}

601 We thank Dr. Xiangyu Liu at Tsinghua University for providing the plasmid expressing Gß1y2.

602 We thank staff at Shuimu BioSciences for their help with cryo-EM data collection. All EM

603 images were collected at Shuimu BioSciences. This work was supported by Chinese

604 Ministry of Science and Technology, Beijing Municipal Science \& Technology Commission

605 (Z201100005320012) and Tsinghua University.

606

607 Author contributions

608 W.J. purified the protein complex, collected cryo-EM data, performed cryo-EM data 609 processing and model building, performed cellular assay with input from S.Z. S.Z. and W.J.

610 wrote the manuscript.

611

612 Competing interests

613 The authors declare no competing interests.

614

615 Data availability

616 The atomic structures have been deposited at the Protein Data Bank (PDB) under the 617 accession codes XXX. The EM maps have been deposited at the Electron Microscopy Data 618 Bank (EMDB) under the accession numbers XXX. 
619 References

620 1. Tatemoto K, Rokaeus A, Jornvall H, McDonald TJ, \& Mutt V (1983) Galanin - a novel

621 biologically active peptide from porcine intestine. FEBS Lett 164(1):124-128.

622 2. Sipkova J, Kramarikova I, Hynie S, \& Klenerova V (2017) The galanin and galanin

623 receptor subtypes, its regulatory role in the biological and pathological functions.

$624 \quad$ Physiol Res 66(5):729-740.

625 3. Lang R, et al. (2015) Physiology, signaling, and pharmacology of galanin peptides

626 and receptors: three decades of emerging diversity. Pharmacol Rev 67(1):118-175.

627 4. Branchek TA, Smith KE, Gerald C, \& Walker MW (2000) Galanin receptor subtypes.

$628 \quad$ Trends Pharmacol Sci 21(3):109-117.

629 5. Wang S \& Gustafson EL (1998) Galanin receptor subtypes. Drug News Perspect $630 \quad 11(8): 458-468$.

631 6. Lin EJ, et al. (2003) Recombinant AAV-mediated expression of galanin in rat 632 hippocampus suppresses seizure development. Eur J Neurosci 18(7):2087-2092.

633 7. Haberman RP, Samulski RJ, \& McCown TJ (2003) Attenuation of seizures and 634 neuronal death by adeno-associated virus vector galanin expression and secretion. $635 \quad$ Nat Med 9(8):1076-1080.

636 8. Millon C, et al. (2019) Role of the galanin N-terminal fragment (1-15) in anhedonia: 637 Involvement of the dopaminergic mesolimbic system. J Psychopharmacol $638 \quad 33(6): 737-747$.

639 9. Li SY, et al. (2017) Involvement of galanin and galanin receptor 1 in nociceptive 
modulation in the central nucleus of amygdala in normal and neuropathic rats. Sci $\operatorname{Rep} 7(1): 15317$

642 10. Kokaia $M$, et al. (2001) Suppressed kindling epileptogenesis in mice with ectopic overexpression of galanin. Proc Natl Acad Sci U S A 98(24):14006-14011.

644 11. Guipponi M, et al. (2015) Galanin pathogenic mutations in temporal lobe epilepsy. Hum Mol Genet 24(11):3082-3091.

646 12. Xu XF, et al. (2016) Galanin and its receptor system promote the repair of injured sciatic nerves in diabetic rats. Neural Regen Res 11(9):1517-1526.

648 13. Elliott-Hunt CR, Pope RJ, Vanderplank P, \& Wynick D (2007) Activation of the galanin receptor 2 (GalR2) protects the hippocampus from neuronal damage. J Neurochem

651 14. Kroeger D, et al. (2018) Galanin neurons in the ventrolateral preoptic area promote sleep and heat loss in mice. Nat Commun 9(1):4129.

653 15. Reichert S, Pavon Arocas O, \& Rihel J (2019) The Neuropeptide Galanin Is Required for Homeostatic Rebound Sleep following Increased Neuronal Activity. Neuron 104(2):370-384 e375.

656 16. Kim DK, et al. (2014) Coevolution of the spexin/galanin/kisspeptin family: Spexin 657 activates galanin receptor type II and III. Endocrinology 155(5):1864-1873.

658 17. Mills EG, Izzi-Engbeaya C, Abbara A, Comninos AN, \& Dhillo WS (2021) Functions of 659 galanin, spexin and kisspeptin in metabolism, mood and behaviour. Nat Rev 
661 18. Kask K, Berthold M, Kahl U, Nordvall G, \& Bartfai T (1996) Delineation of the peptide binding site of the human galanin receptor. $E M B O J 15(2): 236-244$.

663 19. Church WB, Jones KA, Kuiper DA, Shine J, \& lismaa TP (2002) Molecular modelling and site-directed mutagenesis of human GALR1 galanin receptor defines determinants of receptor subtype specificity. Protein Eng 15(4):313-323.

666 20. Nehme R, et al. (2017) Mini-G proteins: Novel tools for studying GPCRs in their active conformation. PLoS One 12(4):e0175642.

668 21. Maeda S, et al. (2018) Development of an antibody fragment that stabilizes GPCR/G-protein complexes. Nat Commun 9(1):3712.

670 22. Smith KE, et al. (1997) Expression cloning of a rat hypothalamic galanin receptor coupled to phosphoinositide turnover. J Biol Chem 272(39):24612-24616.

672 23. Fathi Z, et al. (1997) Cloning, pharmacological characterization and distribution of a novel galanin receptor. Brain Res Mol Brain Res 51(1-2):49-59.

674 24. Carpenter KA, et al. (1999) The glycine residue in cyclic lactam analogues of galanin(1-16)-NH2 is important for stabilizing an N-terminal helix. Biochemistry $38(46): 15295-15304$.

677 25. Barany-Wallje E, Andersson A, Graslund A, \& Maler L (2004) NMR solution structure and position of transportan in neutral phospholipid bicelles. FEBS Lett 567(2-3):265-269 .

680 26. Kruse AC, et al. (2013) Activation and allosteric modulation of a muscarinic acetylcholine receptor. Nature 504(7478):101-106. 
682 27. Shihoya W, et al. (2016) Activation mechanism of endothelin ETB receptor by 683 endothelin-1. Nature 537(7620):363-368.

684 28. Koehl A, et al. (2018) Structure of the micro-opioid receptor-Gi protein complex. Nature 558(7711):547-552.

686 29. Hong C, et al. (2021) Structures of active-state orexin receptor 2 rationalize peptide and small-molecule agonist recognition and receptor activation. Nat Commun 12(1):815

689 30. Floren A, Land T, \& Langel U (2000) Galanin receptor subtypes and ligand binding. Neuropeptides 34(6):331-337.

691 31. Land T, et al. (1991) Linear and cyclic N-terminal galanin fragments and analogs as ligands at the hypothalamic galanin receptor. Int J Pept Protein Res 38(3):267-272.

693 32. Jumper J, et al. (2021) Highly accurate protein structure prediction with AlphaFold. Nature 596(7873):583-589.

695 33. Maeda S, Qu Q, Robertson MJ, Skiniotis G, \& Kobilka BK (2019) Structures of the M1 and M2 muscarinic acetylcholine receptor/G-protein complexes. Science 364(6440):552-557.

698 34. Moro O, Lameh J, Hogger P, \& Sadee W (1993) Hydrophobic amino acid in the i2 loop plays a key role in receptor-G protein coupling. $J$ Biol Chem 268(30):22273-22276.

701 35. Xiao P, et al. (2021) Ligand recognition and allosteric regulation of DRD1-Gs signaling complexes. Cell184(4):943-956 e918. 
703 36. Blakemore LJ \& Trombley PQ (2017) Zinc as a Neuromodulator in the Central 704 Nervous System with a Focus on the Olfactory Bulb. Front Cell Neurosci11:297.

705 37. Kay AR \& Toth K (2008) Is zinc a neuromodulator? Sci Signal 1(19):re3.

706 38. Anderson CT, et al. (2015) Modulation of extrasynaptic NMDA receptors by synaptic and tonic zinc. Proc Natl Acad Sci U S A 112(20):E2705-2714.

708

39. Kalappa BI, Anderson CT, Goldberg JM, Lippard SJ, \& Tzounopoulos T (2015) AMPA receptor inhibition by synaptically released zinc. Proc Natl Acad Sci $U S A$ 112(51):15749-15754.

711 40. Vogt K, Mellor J, Tong G, \& Nicoll R (2000) The actions of synaptically released zinc at hippocampal mossy fiber synapses. Neuron 26(1):187-196.

713 41. Swaminath G, Steenhuis J, Kobilka B, \& Lee TW (2002) Allosteric modulation of beta2-adrenergic receptor by $\mathrm{Zn}(2+)$. Mol Pharmaco/61(1):65-72.

715 42. Holst B, Elling CE, \& Schwartz TW (2002) Metal ion-mediated agonism and agonist enhancement in melanocortin MC1 and MC4 receptors. $J$ Biol Chem 277(49):47662-47670.

718 43. Nunez D, Kumar R, \& Hanahan DJ (1989) Inhibition of [3H]platelet activating factor (PAF) binding by Zn2+: a possible explanation for its specific PAF antiaggregating effects in human platelets. Arch Biochem Biophys 272(2):466-475.

721 44. van der Westhuizen ET, Valant C, Sexton PM, \& Christopoulos A (2015) Endogenous allosteric modulators of $G$ protein-coupled receptors. J Pharmacol Exp Ther 353(2):246-260. 
724 45. Yuan Y, et al. (2021) Structures of signaling complexes of lipid receptors S1PR1 and S1PR5 reveal mechanisms of activation and drug recognition. Cell Res.

726 46. Xu P, et al. (2021) Structures of the human dopamine D3 receptor-Gi complexes. Mol Cel/81(6):1147-1159 e1144.

728 47. Kato HE, et al. (2019) Conformational transitions of a neurotensin receptor 1-Gi1 complex. Nature 572(7767):80-85.

730 48. Liu Q, et al. (2021) Ligand recognition and G-protein coupling selectivity of cholecystokinin A receptor. Nat Chem Biol.

732 49. Krishna Kumar K, et al. (2019) Structure of a Signaling Cannabinoid Receptor 1-G Protein Complex. Cell 176(3):448-458 e412.

734 50. Kim HR, et al. (2020) Structural mechanism underlying primary and secondary 735 coupling between GPCRs and the Gi/o family. Nat Commun 11(1):3160.

736 51. Conklin BR, Farfel Z, Lustig KD, Julius D, \& Bourne HR (1993) Substitution of three 737 amino acids switches receptor specificity of $\mathrm{Gq}$ alpha to that of $\mathrm{Gi}$ alpha. Nature 363(6426):274-276.

739 52. Conklin BR, et al. (1996) Carboxyl-terminal mutations of $\mathrm{Gq}$ alpha and Gs alpha that alter the fidelity of receptor activation. Mol Pharmaco/50(4):885-890.

741 53. Semack A, Sandhu M, Malik RU, Vaidehi N, \& Sivaramakrishnan S (2016) Structural Elements in the Galphas and Galphaq C Termini That Mediate Selective G Protein-coupled Receptor (GPCR) Signaling. J Biol Chem 291(34):17929-17940.

744 54. Zheng S, Abreu N, Levitz J, \& Kruse AC (2019) Structural basis for KCTD-mediated 
rapid desensitization of GABAB signalling. Nature 567(7746):127-131.

746 55. Zheng SQ, et al. (2017) MotionCor2: anisotropic correction of beam-induced motion for improved cryo-electron microscopy. Nat Methods 14(4):331-332.

748 56. Punjani A, Rubinstein JL, Fleet DJ, \& Brubaker MA (2017) cryoSPARC: algorithms for rapid unsupervised cryo-EM structure determination. Nat Methods 14(3):290-296.

750 57. Scheres SH (2012) RELION: implementation of a Bayesian approach to cryo-EM structure determination. J Struct Bio/180(3):519-530.

752 58. Sanchez-Garcia R, et al. (2020) DeepEMhancer: a deep learning solution for cryo-EM volume post-processing. BioRxiv.

754 59. Pettersen EF, et al. (2004) UCSF Chimera--a visualization system for exploratory research and analysis. J Comput Chem 25(13):1605-1612.

756 60. Emsley P \& Cowtan K (2004) Coot: model-building tools for molecular graphics. Acta Crystallogr D Biol Crystallogr 60(Pt 12 Pt 1):2126-2132.

758 61. Adams PD, et al. (2010) PHENIX: a comprehensive Python-based system for macromolecular structure solution. Acta Crystallogr D Biol Crystallogr 66(Pt 2):213-221.

761 62. Inoue A, et al. (2019) Illuminating G-Protein-Coupling Selectivity of GPCRs. Cell 177(7):1933-1947 e1925. 